\title{
Ratiometric Bioluminescent Sensor Proteins Based on Intramolecular Split Luciferase Complementation
}

\author{
Yan Ni, Remco Arts, and Maarten Merkx*i)
}

Laboratory of Chemical Biology and Institute for Complex Molecular Systems, Department of Biomedical Engineering, Eindhoven University of Technology, Den Dolech 2, 5612 AZ Eindhoven, The Netherlands

\section{Supporting Information}

ABSTRACT: Bioluminescent sensor proteins provide attractive tools for applications ranging from in vivo imaging to point-of-care testing. Here we introduce a new class of ratiometric bioluminescent sensor proteins that do not rely on direct modulation of BRET efficiency, but are based on competitive intramolecular complementation of split NanoLuc luciferase. Proof of concept for the feasibility of this sensor principle was provided by developing a blue-red light emitting sensor protein for the detection of anti-HIV1-p17 antibodies with a $500 \%$ change in emission ratio and a $K_{\mathrm{d}}$ of $10 \mathrm{pM}$. The new sensor design also improved the dynamic response of a sensor for the therapeutic antibody cetuximab 4fold, allowing the direct quantification of this anti-EGFR antibody in undiluted blood plasma. The modular sensor architecture allows easy and systematic tuning of a sensor's dynamic range and should be generally applicable to allow rational engineering of bioluminescent sensor proteins.

KEYWORDS: biosensors, BRET, split luciferase, protein engineering, antibodies

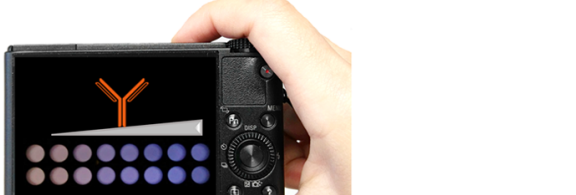

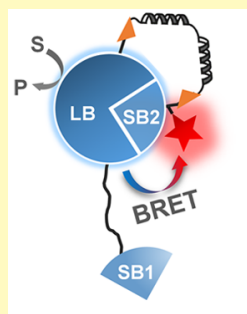
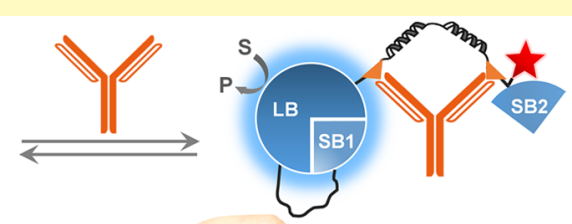

pubs.acs.org/acssensors ptical sensor proteins allow real-time monitoring of molecular events in complex biological environments, with applications ranging from intracellular imaging to pointof-care diagnostics. While fluorescent sensors have become attractive tools for intracellular imaging, their dependence on external illumination has hampered applications in strongly absorbing or scattering media such as blood. ${ }^{1,2}$ This issue has been successfully addressed by employing bioluminescent sensor proteins that use a luciferase to generate light by catalyzing the oxidation of luciferin substrates. ${ }^{3,4}$ The development of bioluminescent sensor proteins that display a robust signal change upon ligand binding remains an important molecular engineering challenge, however. Intensiometric reporter luciferases have been constructed by insertion of a ligand binding domain within a conformationally sensitive part of the enzyme or by ligand-induced complementation of a split luciferase. $^{5-10}$ The first approach can be cumbersome because of its reliance on subtle conformational changes, whereas the more modular split luciferase approach requires at least two sensor parts, rendering its performance concentration dependent. More importantly, both approaches yield intensiometric sensors that are not ideal for quantitative measurements, as their signal intensity depends on many factors including sensor, substrate and product concentration, $\mathrm{pH}$, temperature, and ionic strength.

Ratiometric bioluminescent sensor proteins based on the modulation of bioluminescence resonance energy transfer (BRET) between a luciferase and an acceptor fluorophore are more attractive from this perspective, as in these sensors the ratio of bioluminescence at two different wavelengths is used as a measure of ligand binding. A crucial aspect in the design of robust BRET sensors is the ability to control ligand-induced switching between a high BRET-state, in which luciferase and the fluorescent acceptor are in close proximity, and a low BRET-state in which they are well-separated. Johnsson and coworkers introduced the use of semisynthetic BRET sensor proteins (LUCIDS) in which intramolecular complex formation between a fluorescently labeled ligand analogue and a ligand binding domain is used to create a high BRET state in the absence of analyte. ${ }^{11-14}$ Displacement of the tethered fluorescently labeled ligand by the analyte of interest increases the distance to the luciferase domain fused to the ligand binding, switching the sensor to a low BRET state. Despite the intrinsic modularity of this design, each new receptor domain still requires extensive optimization to ensure sufficient BRET in the off-state of the sensor. An alternative approach reported by our group is the use of so-called helper domains to keep a blue light emitting luciferase domain and a green fluorescent acceptor in close proximity in the off-state. ${ }^{15-17}$ This approach was employed in the development of a sensor platform for antibody detection (LUMABS), in which bivalent binding of a target antibody disrupts the interaction of the helper domains,

Received: November 7, 2018

Accepted: December 11, 2018

Published: December 11, 2018 

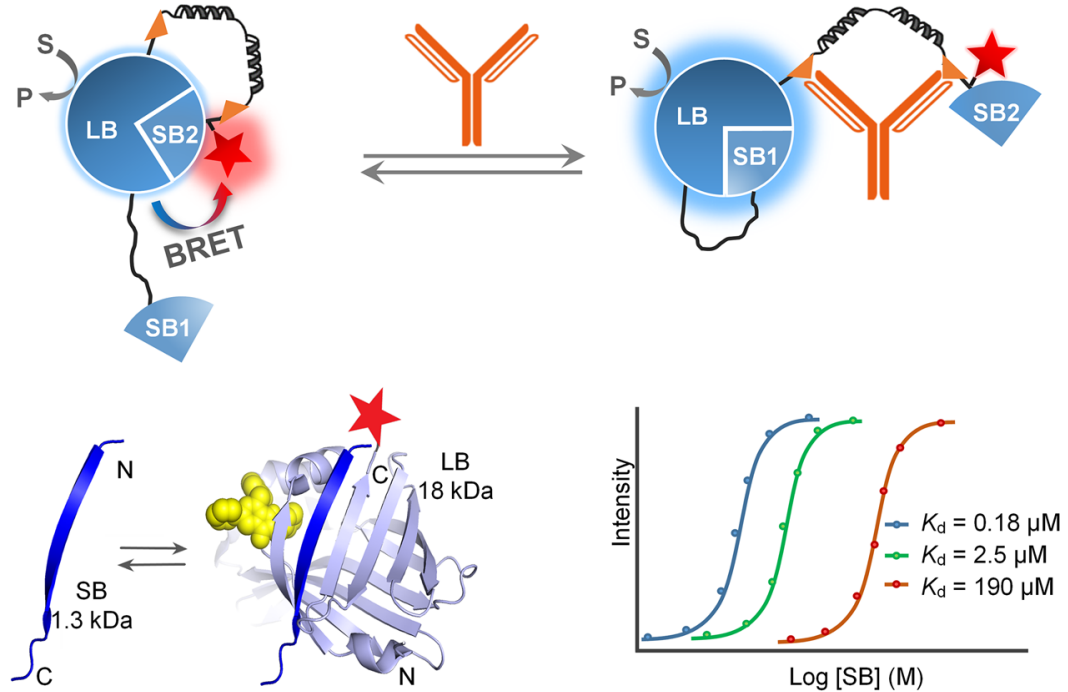

Figure 1. Design of NB-LUMABS. (A) Schematic representation of sensor concept. One large BiT (LB) was fused to two small BiTs (SBs) and a red-emitting fluorophore (red star) is conjugated next to one of the SBs, enabling efficient BRET in the antibody-free state. Bivalent binding of an antibody to the epitopes in the semiflexible linker disrupts the interaction with SB2, changing the color of emission from red to blue. (B) Illustration of split NanoLuc showing the position of SB and the N-terminal Cy3 labeling site. The yellow spheres indicate the proposed binding site $^{18}$ of the furimazine substrate which was docked into the crystal structure of NanoLuc (PDB ID: 5BOU) using AutoDock Vina ${ }^{19}$ and visualized by PyMOL. ${ }^{20}$ (C) Tunability of split NanoLuc interactions. $K_{\mathrm{d}}$ values are not limited to the listed values.
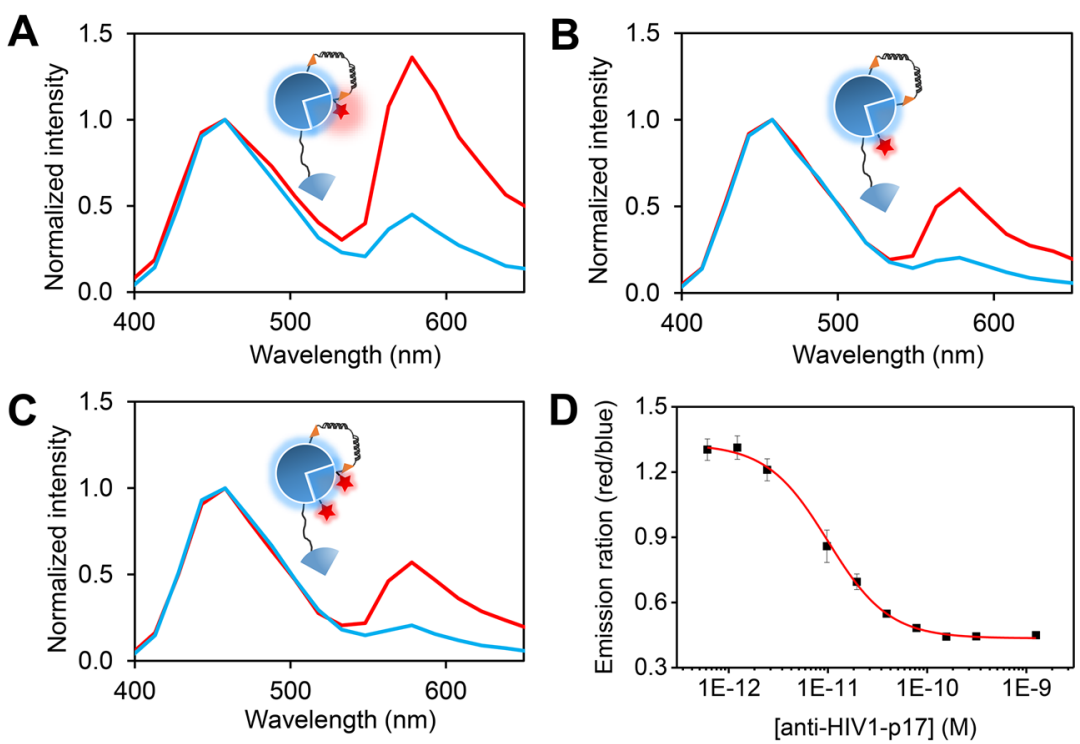

Figure 2. NB-LUMABS for anti-HIV1-p17 antibody. (A-C) Luminescence spectra of 1 pM HIV-NB-LUMABS-1 (A), -2 (B), and -3 (C) in the absence (red line) and presence (blue line) of $1.25 \mathrm{nM}$ anti-HIV1-p17 antibody. (D) Antibody titration to 1 pM of HIV-NB-LUMABS-1 in PBS buffer $(\mathrm{pH} 7.4,1 \mathrm{mg} / \mathrm{mL}$ BSA). Error bars represent mean $\pm \mathrm{SD}(n=3)$. The red line in $(\mathrm{d})$ represents the best fit to eq 1 (SI).

switching the luminescence from green to blue. However, the helper domains and the fluorescent acceptor domain increase the distance between donor luciferase and the acceptor fluorophore, resulting in suboptimal BRET efficiency, in particular for more red-shifted fluorescent acceptors.

Here we introduce a new class of ratiometric bioluminescent sensor proteins that do not rely on modulation of BRET efficiency but are based on competitive intramolecular complementation of split NanoLuc luciferase. NanoLuc is a highly stable and bright, blue light producing luciferase that can be split into two fragments, an $18 \mathrm{kDa}$ large $\mathrm{BiT}(\mathrm{LB})$ and a $1.3 \mathrm{kDa}$ small $\mathrm{BiT}(\mathrm{SB})$, which were designed as a binary complementation reporter to study protein interactions (NanoBiT or $\mathrm{NB}) .^{21,22}$ In our new sensor format (NB-
LUMABS) a single copy of the large fragment (LB) is fused to two copies of the small fragment $(\mathrm{SB})$ yielding a protein switch that can exist in two conformations, where either the $\mathrm{N}$ - or the C-terminal SB binds to the $\mathrm{LB}$ and reconstitutes luciferase activity (Figure 1). A red-emitting fluorophore is conjugated next to one of the SBs, permitting efficient BRET and consequently the emission of red light in only one of the two conformations. Bivalent binding of an antibody disrupts the intramolecular interaction of the fluorescently labeled SB, which allows reconstitution of NanoLuc by the nonfluorescently labeled SB, resulting in a color transition from red to blue. An important property of the split NanoLuc system is that the affinity between the LB and SB can be systematically varied between $K_{\mathrm{d}}=0.7 \mathrm{nM}$ and $K_{\mathrm{d}}=190 \mu \mathrm{M}^{22}$ 
Table 1. Properties of NB-LUMABS Sensors ${ }^{a}$

\begin{tabular}{|c|c|c|c|c|c|c|}
\hline Target & Sensor name & SB1 affinity $^{b}$ & SB2 affinity $^{b}$ & Cy3 position & DR (\%) & Sensor $K_{d}$ \\
\hline \multirow[t]{7}{*}{ Anti-HIV1-p17 } & HIV-NB-LUMABS-1 & $K_{\mathrm{d}}=190 \mu \mathrm{M}$ & $K_{\mathrm{d}}=2.5 \mu \mathrm{M}$ & before SB2 & $218 \pm 12$ & $10.0 \pm 0.5 \mathrm{pM}$ \\
\hline & HIV-NB-LUMABS-2 & $K_{\mathrm{d}}=190 \mu \mathrm{M}$ & $K_{\mathrm{d}}=2.5 \mu \mathrm{M}$ & after SB2 & $177 \pm 4$ & $13.7 \pm 1.3 \mathrm{pM}$ \\
\hline & HIV-NB-LUMABS-3 & $K_{\mathrm{d}}=190 \mu \mathrm{M}$ & $K_{\mathrm{d}}=2.5 \mu \mathrm{M}$ & before and after SB2 & $182 \pm 18$ & $12.1 \pm 0.6 \mathrm{pM}$ \\
\hline & HIV-NB-LUMABS-4 & $K_{\mathrm{d}}=190 \mu \mathrm{M}$ & $K_{\mathrm{d}}=0.18 \mu \mathrm{M}$ & before SB2 & $138 \pm 5$ & $14.2 \pm 4.7 \mathrm{pM}$ \\
\hline & HIV-NB-LUMABS-5 & $K_{\mathrm{d}}=190 \mu \mathrm{M}$ & $K_{\mathrm{d}}=190 \mu \mathrm{M}$ & before SB2 & $252 \pm 15$ & $11.7 \pm 3.7 \mathrm{pM}$ \\
\hline & HIV-NB-LUMABS-6 & $K_{\mathrm{d}}=2.5 \mu \mathrm{M}$ & $K_{\mathrm{d}}=2.5 \mu \mathrm{M}$ & before SB2 & $160 \pm 3$ & $15.2 \pm 1.0 \mathrm{pM}$ \\
\hline & HIV-NB-LUMABS-7 & $K_{\mathrm{d}}=2.5 \mu \mathrm{M}$ & $K_{\mathrm{d}}=0.18 \mu \mathrm{M}$ & before SB2 & $493 \pm 13$ & $11.8 \pm 0.5 \mathrm{pM}$ \\
\hline \multirow[t]{3}{*}{ Cetuximab } & CTX-NB-LUMABS-1 ${ }^{c}$ & $K_{\mathrm{d}}=190 \mu \mathrm{M}$ & $K_{\mathrm{d}}=2.5 \mu \mathrm{M}$ & before SB2 & $233 \pm 12$ & $34.7 \pm 3.7 \mathrm{nM}$ \\
\hline & CTX-NB-LUMABS- $2^{c}$ & $K_{\mathrm{d}}=190 \mu \mathrm{M}$ & $K_{\mathrm{d}}=2.5 \mu \mathrm{M}$ & after SB2 & $110 \pm 3$ & $20.7 \pm 3.4 \mathrm{nM}$ \\
\hline & CTX-NB-LUMABS-3 ${ }^{d}$ & $K_{\mathrm{d}}=190 \mu \mathrm{M}$ & $K_{\mathrm{d}}=2.5 \mu \mathrm{M}$ & before SB2 & $88 \pm 2$ & $189 \pm 16 \mathrm{nM}$ \\
\hline
\end{tabular}

${ }^{a_{T}}$ The measurements were performed in PBS buffer $(\mathrm{pH} 7.4,1 \mathrm{mg} / \mathrm{mL}$ BSA) with sensor concentration of $1 \mathrm{pM}$ for anti-HIV-p17 and $100 \mathrm{pM}$ for cetuximab. ${ }^{b}$ See ref $22 .{ }^{c}$ Meditope sequence CVFDLGTRRLRC (monovalent $K_{\mathrm{d}}=61 \mathrm{nM}$ ). ${ }^{d}$ Meditope sequence CQFDLSTRRLKC (monovalent $\left.K_{\mathrm{d}}=270 \mathrm{nM}\right)$.
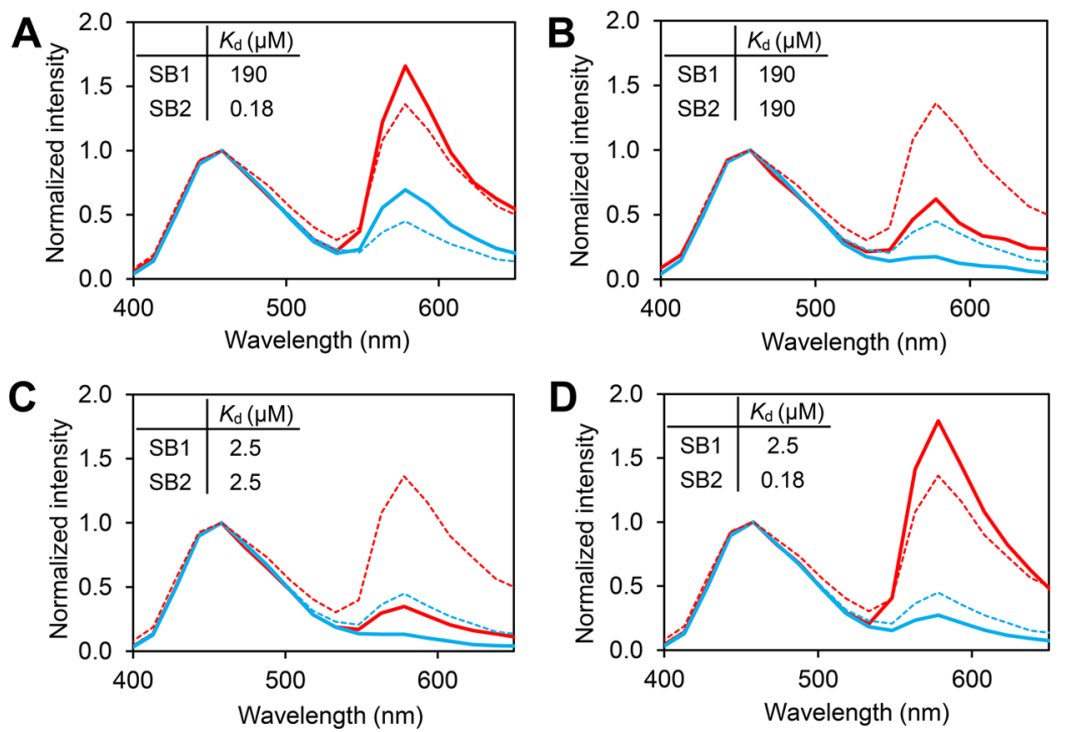

Figure 3. Optimization of sensor response by tuning split NanoLuc interactions. (A-D) Luminescence spectra of 1 pM HIV-NB-LUMABS-4 (A), -5 (B), -6 (C) and -7 (D) in the absence (red solid line) and presence (blue solid line) of $1.25 \mathrm{nM}$ anti-HIV1-p17 antibody. Dashed lines are HIVNB-LUMABS-1 in the absence (red) and presence (blue) of antibody.

allowing precise tuning of the relative thermodynamic stabilities of the two alternative conformations.

To establish proof-of-concept and explore the design constraints for this new sensor concept, we started by developing NB-LUMABS targeting the anti-HIV1-p17 antibody, a well-characterized antibody that binds linear epitopes with high affinity. In our initial design we combined SB1 with a $K_{\mathrm{d}}$ of $190 \mu \mathrm{M}$ and SB2 with a $K_{\mathrm{d}}$ of $2.5 \mu \mathrm{M}$. The higher affinity for SB2 was chosen to favor formation of the high-BRET LBSB2 complex in the absence of antibody, but not too high to prevent activation of the sensor by bivalent antibody binding. SB1 was connected to the N-terminus of $\mathrm{LB}$ via a flexible (GGS $)_{10}$ linker, while SB2 was connected to the C-terminus of LB via a semiflexible linker with the antibody-targeting epitopes at each end of the linker to enable bivalent binding to the target antibody. ${ }^{15}$ The synthetic fluorophore Cy3 was chosen as the BRET acceptor as its emission peak at $565 \mathrm{~nm}$ is well separated from that of NanoLuc. Since BRET efficiency is strongly distance dependent, three constructs were designed with the fluorophore conjugated at different positions via cysteine-maleimide labeling. HIV-NB-LUMABS-1 containing one Cy3 molecule next to the N-terminus of SB2 showed efficient BRET in the initial antibody-free state with the Cy3 peak higher than the NanoLuc emission (Figure 2A), suggesting $\mathrm{Cy} 3$ was kept in close proximity to the substrate binding site of NanoLuc. Sensor variants labeled with either one Cy3 molecule next to C-terminus of SB2 (HIV-NBLUMABS-2) or two Cy3 molecules at both the $\mathrm{N}$ - and $\mathrm{C}$ termini of SB2 (HIV-NB-LUMABS-3) showed a substantially lower $\mathrm{Cy} 3$ emission peak in the absence of antibody (Figure $2 \mathrm{~B}$ and $\mathrm{C})$. The decreased BRET efficiency observed for HIVNB-LUMABS-3 compared to HIV-NB-LUMABS-1 is remarkable and suggests that conjugation of $\mathrm{Cy} 3$ at the $\mathrm{C}$-terminal position may affect the interaction of SB2 with LB. Titration of HIV-NB-LUMABS-1 with increasing concentrations of antiHIV1-p17 antibody revealed a large dynamic range (DR) of $218 \pm 12 \%$ and an overall dissociation constant $\left(K_{d}\right)$ of $10.0 \pm$ $0.5 \mathrm{pM}$ (Figure 2D). Since the dissociation constant for the monovalent antibody-peptide interaction is $42 \mathrm{nM}^{15}$ the bivalent interaction between antibody and the sensor thus resulted in a more than 1000-fold increase in overall affinity.

We next tested whether the already robust ratiometric response could be further enhanced by varying the relative affinities of the split luciferase interactions (Table 1). Increasing the affinity of SB2 from $2.5 \mu \mathrm{M}$ to $0.18 \mu \mathrm{M}$ in HIV-NB-LUMABS-4 resulted in a high emission ratio in the 

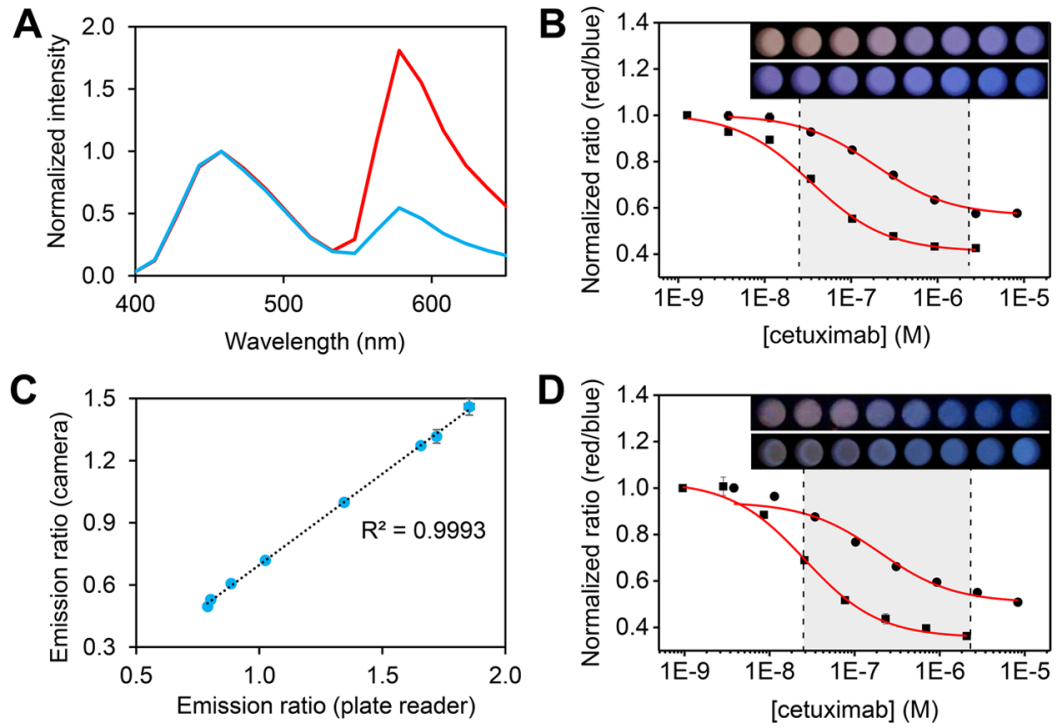

Figure 4. NB-LUMABS for cetuximab. (A) Luminescence spectra of 100 pM CTX-NB-LUMABS-1 in the absence (red line) and presence (blue

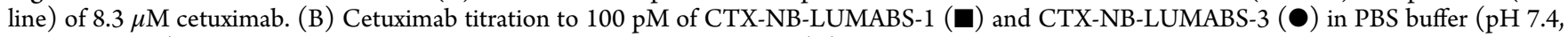
$1 \mathrm{mg} / \mathrm{mL} \mathrm{BSA}$ ). The emission ratios were obtained using a plate reader. (C) Linear regression fit to compare obtained emission ratios of CTXLUMABS-1 in response to cetuximab addition using plate reader and camera measurement. (D) Cetuximab titration to $5 \mathrm{nM}$ of CTX-NBLUMABS-1 ( $)$ and CTX-NB-LUMABS-3 $(\bullet)$ in undiluted blood plasma. The emission ratios were obtained using a plate reader. Error bars represent mean $\pm \mathrm{SD}(n=3)$. Inset: pictures of CTX-NB-LUMABS-1 (top) and CTX-NB-LUMABS-3 (bottom) with varying cetuximab concentration taken by a digital camera. The red lines in B and D represent the best fits to eq 1 (SI). The shaded area represents the physiologically relevant concentration range.

absence of antibody but a moderate ratio change upon addition of antibody (Figure 3A). This result shows that the increased affinity of SB2 further pushes the equilibrium to the high BRET state in the absence of antibody, yet at the same time also hinders bivalent antibody binding to form the low BRET state. Sensor variants in which SB1 and SB2 had the same affinity (HIV-NB-LUMABS-5 and 6, with $K_{\mathrm{d}}$ 's of $190 \mu \mathrm{M}$ and $2.5 \mu \mathrm{M}$, respectively) showed a strong attenuation of the emission ratio in the absence of antibody (Figure 3B,C), suggesting that in this case a substantial fraction resides in the low BRET, SB1-bound state. The optimal combination of SB1 and SB2 affinities was found for HIV-NB-LUMABS-7 (SB1: $K_{\mathrm{d}}$ $=2.5 \mu \mathrm{M}$; SB2: $\left.K_{\mathrm{d}}=0.18 \mu \mathrm{M}\right)$, which combined an increased emission ratio of 1.8 in the absence of antibody, with efficient formation of the low-BRET, bivalent antibody-bound state, yielding a $493 \pm 13 \%$ change in emission ratio (Figure 3D).

Having established the feasibility of using split-NanoLuc complementation as a new sensor principle to generate robust ratiometric bioluminescent proteins with low $\mathrm{pM}$ affinity, we also explored whether the same design principle could be used to improve the performance of LUMABS targeting the clinically important anticancer therapeutic antibody cetuximab. Since cetuximab binds to a discontinuous conformational epitope on the cancer marker EGFR, no linear epitope sequences are available. Nevertheless, we recently identified disulfide-linked cyclic meditope peptides with sufficient affinity to cetuximab and used them to construct a blue-green emitting LUMABS sensor for cetuximab with a relatively modest change in emission ratio $(\mathrm{DR} \sim 60 \%) .{ }^{17,23}$ As the presence of cysteine residues in the meditope peptides precludes the use of cysteine-maleimide chemistry to introduce the Cy3 dye, we instead introduced the noncanonical amino acid paraazidophenylalanine (pAzF) to allow site-specific conjugation with a DBCO-functionalized fluorophore via strain-promoted azide-alkyne click chemistry (SPAAC). In order to incorpo- rate $\mathrm{pAzF}$, a TAG amber stop codon was introduced either before the N-terminus (CTX-NB-LUMABS-1) or after the Cterminus of SB2 (CTX-NB-LUMABS-2) and the anti-HIV1p17 epitope sequences were replaced by the cyclic cetuximab meditope sequences (monovalent $K_{d}$ of $61 \mathrm{nM}$ ). Coexpression with the orthogonal tRNA synthetase/tRNA pair for $\mathrm{pAzF}$ allowed successful incorporation of $\mathrm{pAzF}$ into the cetuximab sensor variants at the desired position. Q-ToF LC-MS analysis of the purified proteins (Figure S2) showed partial reduction of $\mathrm{pAzF}$ to 4-aminophenyl alanine, which limited the degree of Cy3 labeling to 50-60\% (Table S1). Nonetheless, CTX-NBLUMABS-1 still showed bright $\mathrm{Cy} 3$ emission in the absence of cetuximab and a significant decrease in emission ratio upon antibody binding (Figure 4A). A dynamic range of $233 \pm 12 \%$ was achieved, representing a 4-fold improvement compared to the blue-green CTX-LUMABS sensors, ${ }^{17}$ while an overall $K_{\mathrm{d}}$ of $34.7 \pm 3.7 \mathrm{nM}$ obtained from fitting the titration data was found to be similar (Figure 4B). Introduction of $\mathrm{Cy} 3$ at the Cterminus of SB2 in CTX-NB-LUMABS-2 substantially decreased the BRET efficiency in the antibody-free state (Figure S4), which is consistent with the results obtained for HIV-NB-LUMABS-2. We also constructed a sensor variant (CTX-NB-LUMABS-3) containing a cyclic cetuximab meditope with a weaker affinity (monovalent $K_{\mathrm{d}}$ of $270 \mathrm{nM}$ ). Titration experiments with CTX-NB-LUMABS-3 showed a $K_{\mathrm{d}}$ of $189 \pm 16 \mathrm{nM}$, enabling this sensor to reliably measure high cetuximab concentrations (Figure 4B). The lower affinity of CTX-NB-LUMABS-3 for cetuximab also resulted in a more rapid response compared to that of CTX-NB-LUMABS-1 (Figure S5). These results demonstrate that the NB-LUMABS sensor format can be reconfigured to detect antibodies recognizing disulfide constrained cyclic peptides, yielding two cetuximab sensors whose combined responsive regime covers the clinically relevant cetuximab concentration range $(25 \mathrm{nM}$ $-2.3 \mu \mathrm{M}){ }^{24}$ 
As an important step toward point-of-care application, we tested whether the bioluminescent signal could be detected by a simple digital camera instead of a dedicated plate reader. Due to the lower enzymatic activity of NanoBiT compared to intact NanoLuc the bioluminescent intensity of NB-LUMABS is less than that of LUMABS (Figure S6), but its brightness was still sufficient to be detected by a digital camera using only $100 \mathrm{pM}$ of sensor. Moreover, the change in color from red-to-blue is more easily recognized compared to the green-to-blue transition of CTX-LUMABS (Figures 4B and S7). Image analysis software (ImageJ) was used to determine the average intensity in the red and blue color channels and the calculated red/blue ratio was plotted as function of antibody concentration. The $K_{d}$ values determined by camera-based measurements matched well with those obtained using the plate reader (Figure 4C, Table S2). Finally, the performance of NBLUMABS was tested in undiluted blood plasma. Although the absolute intensity of the emitted light was lower in blood plasma than that in buffer, the dynamic ranges and apparent affinities were found to be comparable to those obtained in buffer (Figure 4D, Figure S8, Table S2). Detection of the antibody-dependent color changes using a digital camera again yielded comparable results with plate-reader-based recording (Table S2).

In conclusion, a new class of ratiometric bioluminescent sensors has been developed that uses competitive intramolecular complementation of a split luciferase as an attractive alternative to the classical BRET sensor design. The new sensor format significantly improved the performance of previously reported LUMABS antibody sensors, allowing the use of a red-shifted acceptor and showing a large change in emission ratio to detect picomolar concentrations of antibody directly in solution. The intrinsic modularity of the sensor design not only allows easy and systematic tuning of the sensor's dynamic range and antibody specificity, but also represents a generic design to construct ratiometric bioluminescent sensor proteins for applications ranging from in vivo imaging to developing cheap paper-based point-of-care diagnostics $^{25}$ for a wide range of molecular analytes.

\section{ASSOCIATED CONTENT}

\section{S Supporting Information}

The Supporting Information is available free of charge on the ACS Publications website at DOI: 10.1021/acssensors.8b01381.

Materials and methods and supporting figures (PDF)

\section{AUTHOR INFORMATION}

\section{Corresponding Author}

*E-mail: m.merks@tue.nl.

\section{ORCID $\odot$}

Maarten Merkx: 0000-0001-9484-3882

\section{Present Address}

Remco Arts is currently employed by Promega Benelux B.V.

Notes

The authors declare no competing financial interest.

\section{ACKNOWLEDGMENTS}

This work was supported by an ERC starting grant (ERC2011-StG 280255) and an ERC proof of concept grant (ERC2016-PoC 755471).

\section{REFERENCES}

(1) Arts, R.; Aper, S. J. A.; Merkx, M. Engineering BRET-sensor proteins. Methods Enzymol. 2017, 589, 87-114.

(2) Wang, A.; Feng, J.; Li, Y.; Zou, P. Beyond fluorescent proteins: hybrid and bioluminescent indicators for imaging neural activities. ACS Chem. Neurosci. 2018, 9, 639-650.

(3) Suzuki, K.; Nagai, T. Recent progress in expanding the chemiluminescent toolbox for bioimaging. Curr. Opin. Biotechnol. 2017, 48, 135-141.

(4) Komatsu, N.; Terai, K.; Imanishi, A.; Kamioka, Y.; Sumiyama, K.; Jin, T.; Okada, Y.; Nagai, T.; Matsuda, M. A platform of BRETFRET hybrid biosensors for optogenetics, chemical screening, and in vivo imaging. Sci. Rep. 2018, 8, 1-14.

(5) Binkowski, B.; Fan, F.; Wood, K. Engineered luciferases for molecular sensing in living cells. Curr. Opin. Biotechnol. 2009, 20, 1418.

(6) Binkowski, B. F.; Butler, B. L.; Stecha, P. F.; Eggers, C. T.; Otto, P.; Zimmerman, K.; Vidugiris, G.; Wood, M. G.; Encell, L. P.; Fan, F.; et al. A luminescent biosensor with increased dynamic range for intracellular cAMP. ACS Chem. Biol. 2011, 6, 1193-1197.

(7) Teasley Hamorsky, K.; Ensor, C. M.; Wei, Y.; Daunert, S. A bioluminescent molecular switch for glucose. Angew. Chem., Int. Ed. 2008, 47, 3718-3721.

(8) Yang, L.; Nasu, Y.; Hattori, M.; Yoshimura, H.; Kanno, A.; Ozawa, T. Bioluminescent probes to analyze ligand-induced phosphatidylinositol 3,4,5-trisphosphate production with split luciferase complementation. Anal. Chem. 2013, 85, 11352-11359.

(9) Kim, S. B.; Otani, Y.; Umezawa, Y.; Tao, H. Bioluminescent indicator for determining protein-protein interactions using intramolecular complementation of split click beetle luciferase. Anal. Chem. 2007, 79, 4820-4826.

(10) Ohmuro-Matsuyama, Y.; Ueda, H. Homogeneous noncompetitive luminescent immunodetection of small molecules by ternary protein fragment complementation. Anal. Chem. 2018, 90, 3001-3004.

(11) Griss, R.; Schena, A.; Reymond, L.; Patiny, L.; Werner, D.; Tinberg, C. E.; Baker, D.; Johnsson, K. Bioluminescent sensor proteins for point-of-Care therapeutic drug monitoring. Nat. Chem. Biol. 2014, 10, 598-603.

(12) Xue, L.; Yu, Q.; Griss, R.; Schena, A.; Johnsson, K. Bioluminescent antibodies for point-of-care diagnostics. Angew. Chem., Int. Ed. 2017, 56, 7112-7116.

(13) Hiblot, J.; Yu, Q.; Sabbadini, M. D. B.; Reymond, L.; Xue, L.; Schena, A.; Sallin, O.; Hill, N.; Griss, R.; Johnsson, K. Luciferases with tunable emission wavelengths. Angew. Chem., Int. Ed. 2017, 56, 14556-14560.

(14) Yu, Q.; Xue, L.; Hiblot, J.; Griss, R.; Fabritz, S.; Roux, C.; Binz, P. A.; Haas, D.; Okun, J. G.; Johnsson, K. Semisynthetic sensor proteins enable metabolic assays at the point of care. Science 2018, $361,1122-1126$

(15) Arts, R.; Den Hartog, I.; Zijlema, S. E.; Thijssen, V.; Van Der Beelen, S. H. E.; Merkx, M. Detection of antibodies in blood plasma using bioluminescent sensor proteins and a smartphone. Anal. Chem. 2016, 88, 4525-4532.

(16) Arts, R.; Ludwig, S. K. J.; Van Gerven, B. C. B.; Estirado, E. M.; Milroy, L. G.; Merkx, M. Semisynthetic bioluminescent sensor proteins for direct detection of antibodies and small molecules in solution. ACS Sensors 2017, 2, 1730-1736.

(17) van Rosmalen, M.; Ni, Y.; Vervoort, D. F. M.; Arts, R.; Ludwig, S. K. J.; Merkx, M. Dual-color bioluminescent sensor proteins for therapeutic drug monitoring of antitumor antibodies. Anal. Chem. 2018, 90, 3592-3599.

(18) Tomabechi, Y.; Hosoya, T.; Ehara, H.; Sekine, S. I.; Shirouzu, M.; Inouye, S. Crystal structure of nanoKAZ: the mutated $19 \mathrm{kDa}$ component of oplophorus luciferase catalyzing the bioluminescent reaction with coelenterazine. Biochem. Biophys. Res. Commun. 2016, 470, 88-93.

(19) Trott, O.; Olson, A. J. AutoDock Vina: improving the speed and accuracy of docking with a new scoring function, efficient 
optimization and multithreading. J. Comput. Chem. 2009, 31, 455461.

(20) PyMOL Molecular Graphics System, v 1.8 Schrödinger, LLC.

(21) Hall, M. P.; Unch, J.; Binkowski, B. F.; Valley, M. P.; Butler, B. L.; Wood, M. G.; Otto, P.; Zimmerman, K.; Vidugiris, G.; MacHleidt, T.; et al. Engineered luciferase reporter from a deep sea shrimp utilizing a novel imidazopyrazinone substrate. ACS Chem. Biol. 2012, 7, 1848-1857.

(22) Dixon, A. S.; Schwinn, M. K.; Hall, M. P.; Zimmerman, K.; Otto, P.; Lubben, T. H.; Butler, B. L.; Binkowski, B. F.; MacHleidt, T.; Kirkland, T. A.; et al. NanoLuc complementation reporter optimized for accurate measurement of protein interactions in cells. ACS Chem. Biol. 2016, 11, 400-408.

(23) van Rosmalen, M.; Janssen, B. M. G.; Hendrikse, N. M.; van der Linden, A. J.; Pieters, P. A.; Wanders, D.; de Greef, T. F. A.; Merkx, M. Affinity maturation of a cyclic peptide handle for therapeutic antibodies using deep mutational scanning. J. Biol. Chem. 2017, 292, 1477-1489.

(24) Azzopardi, N.; Lecomte, T.; Ternant, D.; Boisdron-Celle, M.; Piller, F.; Morel, A.; Gouilleux-Gruart, V.; Vignault-Desvignes, C.; Watier, H.; Gamelin, E.; et al. Cetuximab pharmacokinetics influences progression-free survival of metastatic colorectal cancer patients. Clin. Cancer Res. 2011, 17, 6329-6337.

(25) Tenda, K.; van Gerven, B.; Arts, R.; Hiruta, Y.; Merkx, M.; Citterio, D. Paper-based antibody detection devices using bioluminescent BRET-switching sensor proteins. Angew. Chem., Int. Ed. 2018, 57, 15369-15373. 